\title{
Deploying Medical Students During the COVID-19 Pandemic
}

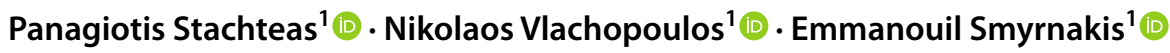

Accepted: 19 August 2021 / Published online: 26 August 2021

(C) International Association of Medical Science Educators 2021

\begin{abstract}
During the novel coronavirus pandemic outbreak, medical education has been inevitably disrupted, while the clinical exposure of many medical students has been hindered. The current article investigates the student role in this large-scale health crisis, in terms of providing for the continuity of medical training and addressing the growing needs of healthcare systems. By presenting different medical education policies implemented worldwide, new perspectives on student involvement are being illuminated. Even during pandemics, students should be motivated to actively serve. The formation of a clear framework for students' roles enhances the preparedness of the medical education community for related future challenges.
\end{abstract}

Keywords Pregraduate/Medical education $\cdot$ Primary care $\cdot$ Medical humanities $\cdot$ Interdisciplinary medical education

\section{Introduction}

The novel Coronavirus SARS-CoV-2, responsible for the COVID-19 disease, emerged from Wuhan, but rapidly spread geographically, affecting millions of people worldwide. This health crisis poses an unprecedented challenge for medical education. Medical educators across the globe have been forced to immediately generate distance learning environments by converting, in a few weeks, the majority of face-to-face theoretical sessions to online sessions [1-3]. Although current evolving web-based ventures can hostand therefore substitute- the majority of didactic lectures of undergraduate curriculum, student clinical exposure remains an indispensable part of medical education, especially in final years for their transition from medical students to junior doctors. Thus, it is reasonable for education providers to wonder: what could be done to narrow the time gap for students who would have been assigned to clinical placements under normal circumstances but who are now waiting?

The aim of this article is to investigate the active role that medical students can have in this large-scale health crisis caused by the recent COVID-19 pandemic, in terms of providing for the continuity of their training and addressing the growing needs of the healthcare system. We also attempt to

Emmanouil Smyrnakis

smyrnak@auth.gr

1 Laboratory of Primary Health Care, General Practice and Health Services Research, School of Medicine, Aristotle University of Thessaloniki, Thessaloniki, Greece highlight the different medical education policies that were implemented worldwide, with the purpose to elucidate the necessary conditions and present an algorithmic framework for the students' possible involvement in clinical and/or outpatient settings.

\section{Students' Possible Engagement During Pandemic}

\section{Early Graduation of Medical Students}

It is essential to gain clarity regarding the range of responsibilities students can receive. Since there are not any clear predictions on how the pandemic will affect the national healthcare systems, there are rising concerns on last year medical students' early-graduation and employment, especially in countries with a high number of COVID-19 cases. In the current pandemic, many medical schools are graduating final-year medical students earlier on the condition that they serve as frontline clinicians. Specifically in Italy, the first European country to be hit hard by the pandemic, medical school graduates skipped the final exams and got sent directly into health service to help fight COVID-19, along with newly hired medical staff and retired doctors, to cover the chronic shortage of healthcare workers [4]. Moreover, the University of Massachusetts Medical School expedited the graduation 2 months earlier than previously scheduled, in order to deploy them to care for patients and alleviate workforce shortages [5]. Worldwide, general dissidence and 
uncertainty has led to varying attitudes among institutions regarding student involvement in pandemic management, but it seems that there are plenty of options-apart from early graduation - for engagement in both inpatient and outpatient activities.

\section{Engaging Students in Inpatient Activities}

Undoubtedly, the direct involvement of students with COVID-19 patients is rather controversial, especially for safety and ethical reasons. Nevertheless, as access to PPE and supervision improve, their contribution could be restricted to low-acuity COVID-19 patients [3]. This includes working in screening and assessment clinics, assisting in GP practices, and triaging in Emergency Departments (EDs) [6, 7]. In Norwich Medical School in the UK, for example, after the announcement of closure, including cancellation of clinical placements, medical students were recruited in areas of greatest clinical need in order to assist with tasks such as basic nursing care, proning, and swab testing with significant benefits to both students and clinical teams [8]. These tasks are more close to their level of competency since senior medical students are clinically experienced and should be proficient at obtaining medical histories, initiating documentation, monitoring, triaging, and providing basic, urgent care for patients with symptoms of COVID-19 [9]. Students can provide a range of duties administrative in nature, such as patient clerking, blood drawing, and phlebotomy, or clinically supportive in nature, such as critical care monitoring in ICUs after specific training [1]. In Denmark, some medical students initiated fasttrack courses in ventilator therapy and nursing assistance and were soon voluntarily deployed to pandemic EDs [10]. Clinical duties were similarly assigned to medical students in Switzerland and in France, who supported frontline workers in many COVID-19 tasks including triaging and massive testing not only in ED and resuscitation units, but also in primary care settings $[11,12]$.

Alternatively, if PPE supplies remained limited, students could provide care to patients treated for non-COVID19related diseases [3]. During a pandemic, patients with other acute or chronic conditions still need ongoing care and regular follow-up. In such circumstances, students can serve by taking histories, participating in common daily procedures, providing patient education, and documenting visits [9]. At 'Sotiria' Athens General Hospital, students responded with excitement and were voluntarily engaged not only in COVID-19 cases, but also in patients with chronic diseases by carrying out useful tasks for frontline providers [13].

\section{Engaging Students in Primary Care Activities}

In primary care activities, medical students could take a variety of responsibilities. One possibility is to help patient temperature and saturation screening or take swabs and implement rapid testing that informs community surveillance $[7,11]$. It has been suggested that 'they can monitor patients with mild COVID-19 symptoms who are not admitted; expedite care for admitted patients by reviewing charts, drafting notes, and ensuring tests are performed; and follow-up with patients after discharge' [9]. Students can also play a pivotal role in the global vaccination campaign against COVID-19. By shouldering various organisational tasks and actively contributing to the history taking and vaccination procedure, they could unburden the medical staff and upgrade the quality of the healthcare facilities offered in the vaccination centres, contributing at the same time to the promotion of their education (improving their clinical, communication and soft skills) and the formation of their professional identity.

In fact, senior medical students are experienced enough to effectively receive many of the responsibilities of a general practitioner (GP) in the community. Cuba implemented a promising prevention plan based on active screening, to interrupt the transmission chain; senior medical students, well-educated in Primary Care and epidemiological principles, moved literally from doorto-door to increase community surveillance and enhance case detection [14]. In addition, public health officials across Canada have begun recruiting senior medical students to assist in the necessary task of tracking down individuals who have been in contact with infected patients [15]. In Denmark, medical students were employed by the Dutch Blood Bank, so as to call potential donors of convalescent plasma, as a possible treatment for patients with COVID-19 [6].

\section{Engaging Students in Public Health Initiatives}

A wider role that medical students can play is related to public health awareness initiatives by offering guidance and challenging false news or misconceptions. For instance, they could positively help influence behaviours via social media by promoting adherence to physical separation and safe travelling. In the USA, students immediately reacted by creating a response team, which promoted public health awareness and was alert for every opportunity to contribute safely [16]. In Brazil, students volunteered in creating an epidemiological information bulletin, which rapidly evolved into a successful platform of public health promotion [17]. 
Pairing students with clinical researchers to serve as expert researchers who obtain the most up-to-date information on protocols and recommendations for overworked clinical staff could be extremely useful and educational [18]. Students from Canada and Greece participated in such interdisciplinary scientific teams by literature briefing and distributing scientific information $[13,19]$. At the Donald and Barbara Zucker School of Medicine, a 2-week synchronous online course was developed to report students on lessons learned from previous pandemics, to discuss ethical issues regarding COVID-19 clinical trials, and to interact with experienced healthcare professionals who provided patient care in the HIV and COVID19 pandemic [20]. Interestingly, an anonymous survey conducted by the end of this course showed that students gained better insight into the COVID-19 pandemic and the research over the possible interventions, and found themselves capable of offering well-grounded guidance to their community, psychologically supporting patients affected from the virus, and making good use of the lessons learned from previous pandemics to enhance their preparedness for future challenges.

In medical schools across the USA, the UK, and other countries, students are volunteering in call centres (e.g., by following up COVID-19 patients with mild symptoms which were advised to stay isolated at home), creating and delivering patient education/information material, collecting masks for local hospitals, and they are also helping with various activities (e.g., childcare for occupied medical staff, logistical assistance, shopping, and collecting prescriptions for people unable to leave their homes) $[2,7,16]$. For example, at the University of Nevada, Reno School of Medicine, medical students were voluntarily employed in a multicounty COVID-19 hotline clinical experience by providing counselling and education via telehealth, so as to adress the new challenges and to assist the underserved rural populations of northern Nevada [21]. Last but not least, in Greece, students volunteered to work hotlines at the two biggest reference centres of the country, and distributed vital information among hospitals and individuals [13].

\section{Discussion and Suggested Pathways}

Despite widespread fear and uncertainty, history may provide us with valuable lessons about medical education during pandemics. During the Spanish influenza emergence of 1918, medical students in Spain, Minnesota, and Philadelphia were actively recruited to replace physicians lost to infection, and in the 1952 polio epidemic in Denmark students were tasked with manually ventilating patients [22-25]. More recently, the severe acute respiratory syndrome (SARS) outbreak of 2003 disrupted medical education (cessation of clinical clerkships and electives, delays in exams) in many countries including China and Canada, but substantial roles were attributed to students in order to provide their valuable help [26-28]. Considerations and suggestions about safe continuity in medical education were also expressed in Korea after the 2012 Middle East respiratory syndrome (MERS) outbreak [29].

Despite these paradigms, it is widely perceived that the student role in response to such unprecedented circumstances is far from clear. COVID-19 revealed curricular gaps on undergraduate medical education in exploring historical pandemics, how to critically integrate scientific literature and square it with the lay press, and how to grapple with emerging ethical issues [20]. Sure is that medical training cannot be halted in every threat of a pandemic, and the medical education community is obliged to organise coordinated responses. Medical students volunteering initiatives is a viable solution for such disruptions and the patient contact opportunities it offers may well complement remote or simulated learning [30]. Nevertheless, the idea of a single holistic solution for all cases seems rather utopian. A more reasonable alternative would be the adoption of practices related to the prevailing conditions of each country.

Naturally, this individualised decision for student involvement should depend on specific criteria. It is of paramount importance that the advantages of such a decision should outweigh the risks so that the cost-benefit ratio will be truly beneficial not only for the overall crisis management, but also for medical students' safety, education and professionalism. This condition gains even bigger value in the crucial point when medical demand outpaces medical capacity [3]. Indeed, if COVID-19 overwhelms the healthcare system's capacity to provide excellent, comprehensive patient care, students should have the opportunity to massively contribute to this fight. But even when medical capacity is adequate, their desire to participate combined with the invaluable educational opportunity make their clinical involvement a reasonable solution as long as occupational safety protocols are observed.

Figure 1 summarises the possible roles that medical students can play depending on the situation of the country in relation to the pandemic outbreak. There are two possible situations during the crisis: (a) pandemic out of control with increased direct needs in healthcare personnel and (b) pandemic in a controlled situation with a relatively adequate frontline human workforce. Subsequently, two distinct categories of each case are presented, depending on the adequacy of the PPE; this is reasonable as student involvement with suspected or confirmed cases calls for additional PPE, shortages of which have already been noted worldwide [1, 3, 4] (Fig. 1). 
Fig. 1 Medical students' possible roles depending on the situation of the country in relation to the pandemic outbreak

\section{PPE adequacy}

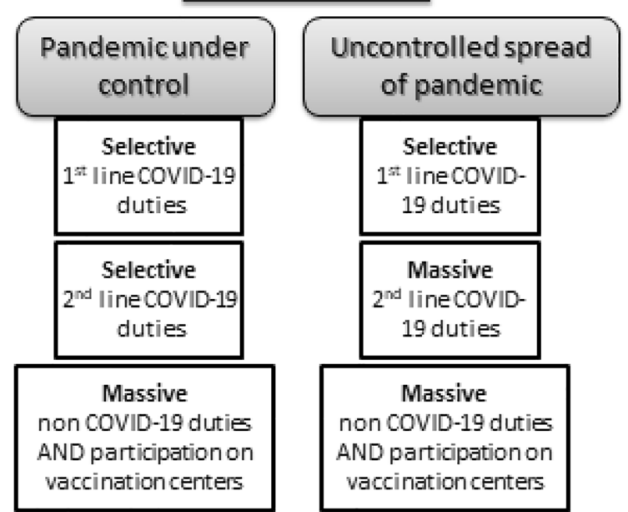

COVID-19 duties 1 st line Engagement on stable low-acuity COVID-19 patients

-ED triaging [i.e. temperature screening], taking swabs

- Patient monitoring

- Clinically supportive duties [e.g. blood

sampling, nursing assistance]

-Spread surveillance and early case

detection

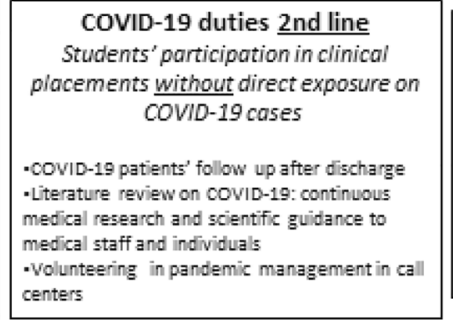

PPE shortage

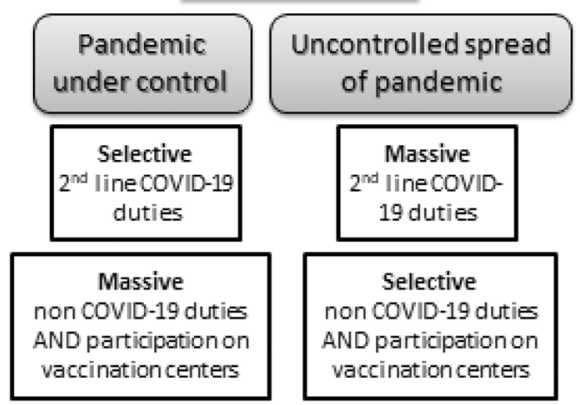

There are two questions that need to be answered before the involvement of medical students. Is it safe for students? And if so, is students' assistance necessary for the healthcare system? A massive response refers to the mobilisation of the majority of medical students when supplies are adequate and the healthcare system's needs are augmented, while the selective response is related to the partial voluntary engagement of a small portion of students when supplies are limited (safety and ethical considerations) or pandemic spread is under control (student assistance is not necessary). Student involvement with active COVID-19 cases should always be selective, even in a controlled situation with an abundance of protective equipment.

In addition to the adequacy of PPE, another key parameter that influences the students' final roles is the capacity for supervision. In burdened countries with a growing number of cases, it is considered that the possibility of supervision by frontline staff is limited due to the increased workload and possible selfisolation or illness. An interesting alternative is engaging retired healthcare professionals who can contribute to student training either in-person or remotely. Quarantined doctors can also contribute to distance education. Training and supervision in COVID19-related structures can take place either by employees in these structures or in the form of peer education [2].

In this article, we endeavoured to describe some of the attempts made to substitute medical education during the current COVID-19 pandemic. Our algorithmic approach is based on the heterogeneous experiences recorded in many countries across the globe and, therefore, can serve as a useful starting point in the formation of broader guidelines regarding medical student pandemic response strategies. Each country is affected diversely by the pandemic, every prospective local or global pandemic will probably have different epidemiological characteristics, population health needs and medical training objectives vary down the stream of time, and the medical community has different options in its armamentarium. Consequently, not every option is available or replicable in every situation, but measures should be individualised according to the students' needs and the national healthcare systems' capacities.

The outbreak of a large-scale contagious, infectious disease inevitably hampers inpatient medical education. Especially in the early stages of a pandemic, when parameters such as viral contagion and mode of transmission are not fully understood, the timing seems improper for student involvement for safety and ethical reasons. Nevertheless, a pandemic poses a great learning opportunity for students in tackling a health crisis. Concurrently, student contribution seems to be precious for an overburdened healthcare system. Therefore, the option to voluntarily deploy students, mainly in outpatient positions and primary care settings, but also in direct patient (COVID and non-COVID) activities, should be promoted under specific conditions in the proper timing and cannot be rejected in advance. The formation of a clear framework for students' roles in such circumstances enhances the preparedness of the medical education community for related future challenges in order to maintain sound and robust training for future physicians. 
Acknowledgements We thank Emily Fisher, MBA, Impact Officer and Founding Team member of the Global Shapers Thessaloniki Hub, an initiative of the World Economic Forum, who provided language and editing services.

Author Contribution All authors contributed to the study conception and design. Material preparation, data collection, and analysis were performed by Panagiotis Stachteas and Nikolaos Vlachopoulos, who also communally wrote the first draft of the manuscript and revised previous versions of the manuscript. The senior author Emmanouil Smyrnakis read and approved the final manuscript.

\section{Declarations}

Ethics Approval Not necessary/not applicable.

Consent to Participate Not applicable.

Conflict of Interest The authors declare no competing interests.

\section{References}

1. Arandjelovic A, Arandjelovic K, Dwyer K, Shaw C. COVID-19: considerations for medical education during a pandemic. MedEdPublish. 2020;9(1):87.

2. Rose S. Medical student education in the time of COVID-19. JAMA. 2020;323(21):2131-2.

3. Stokes DC. Senior medical students in the COVID-19 response: an opportunity to be proactive. Acad Emerg Med. 2020;27(4):343-5.

4. Paterlini M. On the front lines of coronavirus: the Italian response to covid-19. BMJ. 2020;368:m1065.

5. Flotte TR, Larkin AC, Fischer MA, Chimienti SN, Demarco DM, Fan PY, Collins MF. Accelerated graduation and the deployment of new physicians during the COVID-19 pandemic. Acad Med. 2020;95(10):1492-4.

6. Bank I, Wijnen-Meijer M. Why should medical students (not) be recruited to care for patients with COVID-19? BMC Med Educ. 2020;20(1):34.

7. Blom IM, Whyatt G, Viva I, Martin A. The worldwide contribution of medical students during the COVID-19 pandemic: quantitative and qualitative research. Research Square. 2020. https://doi.org/ 10.21203/rs.3.rs-25384/v1.

8. Maas K, Wassef M, Kulkarni M, Bowker L. Employment of medical and physician associate students in an NHS trust during the COVID19 pandemic. Future Healthc J. 2021;8(1):e146-9.

9. Miller DG, Pierson L, Doernberg S. The role of medical students during the COVID-19 pandemic. Ann Intern Med. 2020;173(2):145-6.

10. Rasmussen S, Sperling P, Poulsen MS, Emmersen J, Andersen $\mathrm{S}$. Medical students for health-care staff shortages during the COVID-19 pandemic. Lancet. 2020;395(10234):e79-80.

11. Klasen JM, Meienberg A, Nickel C, Bingisser R. SWAB team instead of SWAT team: medical students as a frontline force during the COVID-19 pandemic. Med Educ. 2020;54(9):860.

12. Pelaccia T, Sibilia J, Fels É, et al. And if we had to do it all over again, would we send medical students to the emergency departments during a pandemic? Lessons learned from the COVID-19 outbreak [published online ahead of print, 2021 Jan 16]. Intern Emerg Med. 2021;1-8.

13. Vlachopoulos N, Smyrnakis E, Stachteas P, Exindari M, Gioula G, Papa A. Medical students during COVID-19 pandemic: lessons learned from response teams in Greece. Int J Med Students. 2020;8(2):191-3.

14. Gorry C. COVID-19 case detection: Cuba's active screening approach. MEDICC Rev. 2020;22(2):58-63.

15. Statement of need program for medical graduates pursuing postgraduate medical training in the US. Government of Canada. 2020. https://www.canada.ca/en/healthcanada/services/ health-care-system/health-humanresources/statements-needpostgraduate-medicaltraining-united-states.html. Accessed 17 Oct 2020.

16. Soled D, Goel S, Barry D, Erfani P, Joseph N, Kochis M, Uppal $\mathrm{N}$, Velasquez D, Vora K, Scott KW. Medical student mobilization during a crisis: lessons from a COVID-19 medical student response team. Acad Med. 2020;95(9):1384-7.

17. Villela EFM, De Oliveira FM, Leite ST, Bollela VR. Student engagement in a public health initiative in response to COVID19. Med Educ. 2020;54(8):763-4.

18. Khamees D, Brown CA, Arribas M, Murphey AC, Haas MRC, House JB. In Crisis: Medical Students in the COVID-19 Pandemic. AEM Educ Train. 2020;4(3):284-90.

19. Boodman C, Lee S, Bullard J. Idle medical students review emerging COVID-19 research. Med Educ Online. 2020;25(1):1770562.

20. Willey JM, Olvet DM, Bird JB, Brenner JM. Pandemics past and present: a guided inquiry approach. J Med Educ Curric Dev. 2020;7:2382120520976957.

21. Carson S, Peraza LR, Pucci M, Huynh J. Student hotline improves remote clinical skills and access to rural care. PRiMER. 2020;4:22.

22. West JB. The physiological challenges of the 1952 Copenhagen poliomyelitis epidemic and a renaissance in clinical respiratory physiology. J Appl Physiol. 2005;99(2):424-32.

23. Starr I. Influenza in 1918: recollections of the epidemic in Philadelphia. Ann Intern Med. 2006;145(2):138-40.

24. Trilla A, Trilla G, Daer C. The 1918 "Spanish Flu" in Spain. Clin Infect Dis. 2008;47(5):668-73.

25. Ott M, Shaw SF, Danila RN, Lynfield R. Lessons learned from the 1918-1919 influenza pandemic in Minneapolis and St. Paul, Minnesota. Public Health Rep. 2007;122(6):803-810.

26. Patil NG, Chan Y, Yan H. SARS and its effect on medical education in Hong Kong. Med Educ. 2003;37(12):1127-8.

27. Rieder MJ, Salvadori M, Bannister S, Kenyon C. Collateral damage: the effect of SARS on medical education. Clin Teach. 2004;1(2):85-9.

28. Clark J. Fear of SARS thwarts medical education in Toronto. BMJ. 2003;326(7393):784.

29. Park SW, Jang HW, Choe YH, et al. Avoiding student infection during a Middle East respiratory syndrome (MERS) outbreak: a single medical school experience. Korean J Med Educ. 2016;28(2):209-17.

30. Chawłowska E, Staszewski R, Lipiak A, et al. Student volunteering as a solution for undergraduate health professions education: lessons from the COVID-19 pandemic. Front Public Health. 2021;8:633888

Publisher's Note Springer Nature remains neutral with regard to jurisdictional claims in published maps and institutional affiliations. 